\title{
Analysis of Asymmetries in Ictal and Inter-Ictal SPECT Images for the Localization of Epileptic Foci
}

\author{
Dorit Merhof, Christian Mathers, Thomas Wright, Torsten Kuwert, and Günther Platsch
}

\begin{abstract}
In patients suffering from focal epilepsy, the region of seizure onset is characterized by hyperperfusion during the seizure (ictal state), and hypoperfusion in normal (inter-ictal) state. For diagnosis and surgery planning in epilepsy patients, ictal and inter-ictal SPECT images play a major role. However, comparison of this kind of data is a difficult clinical problem due to varying physiological uptake in brain regions. For this reason, different analysis methods such as standard reading by a clinical expert, comparison to a normal database, subtraction ictal SPECT co-registered to MRI (SISCOM) or asymmetry analysis are applied, for a comprehensive analysis of the data. The latter is usually hampered by functional and anatomical asymmetries which aggravate analysis. For this reason, a novel approach for asymmetry analysis is presented in this work, which overcomes the aforementioned limitations. The approach was successfully applied to SPECT datasets of 10 epilepsy patients.
\end{abstract}

\section{INTRODUCTION}

$\mathbf{E}$ PILEPSY is one of the most common neurological disorders in children and young adults. In patients not responding to medical treatment, epilepsy surgery may be considered to control seizures, in case of a focal epilepsy. A major goal in surgery planning for epilepsy patients is to localize the epileptic focus. For this purpose, standard approaches such as magnetic resonance imaging (MRI) and electroencephalography (EEG) recordings are applied. Additionally, molecular imaging based on single photon emission computed tomography (SPECT) plays a major role in localizing the epileptic focus.

SPECT makes it possible to study cerebral blood flow $(\mathrm{CBF})$, which can be quantified as a rate of tissue perfusion, expressed as volume of blood flowing through a given quantity of tissue per unit of time. In patients suffering from focal epilepsy, the region of seizure onset is characterized by hyperperfusion during the seizure (ictal state), and hypoperfusion in normal (inter-ictal) state. Both an ictal and inter-ictal SPECT scan are acquired using the tracer ${ }^{99 \mathrm{~m}} \mathrm{Tc}-\mathrm{ECD}$ or ${ }^{99 \mathrm{~m}} \mathrm{Tc}-$ HMPAO, which are effective markers of cerebral perfusion imaging in epilepsy [1].

The analysis of ictal and inter-ictal SPECT images is a challenging problem. A conventional side-by-side comparison of the scans is hampered by different patient position, varying

D. Merhof is with the University of Konstanz, Konstanz, Germany.

C. Mathers is with Siemens Molecular Imaging, Oxford, UK.

T. Wright is with Siemens Molecular Imaging, Oxford, UK.

T. Kuwert is with the Clinic of Nuclear Medicine, University of ErlangenNuremberg, Erlangen, Germany.

G. Platsch is with Siemens Molecular Imaging EU, Erlangen, Germany. intensities of the scans and the lack of clear anatomical information. The most challenging problem however arises from increased/reduced uptake in regions other than the epileptic focus due to normal brain function, e.g., in the visual cortex or the motor cortex.

For a comprehensive analysis of the data, different approaches are therefore needed, and different analysis methods such as standard reading by a clinical expert, comparison to a normal database [2], subtraction ictal SPECT co-registered to MRI (SISCOM) [3], [4] or asymmetry analysis [5] are applied for a comprehensive analysis of the data.

The latter is usually hampered by asymmetries due to varying functional uptake between both brain hemispheres. Also, since the brain hemispheres are not perfectly symmetric, anatomical differences occur as well. In this work a novel technique for asymmetry analysis is presented, which is able to account for these effects.

\section{Material And Methods}

\section{A. Image Data}

The ${ }^{99 \mathrm{~m}} \mathrm{Tc}-\mathrm{ECD}$ SPECT datasets used in this work comprise 10 patients with mixed epilepsy (age 17 to 44 , mean age 30, 6 male, 4 female) and were acquired at the Clinic of $\mathrm{Nu}$ clear Medicine, University of Erlangen-Nuremberg, Erlangen, Germany.

For the acquisition of the inter-ictal scans, injection of 9$10 \mathrm{MBq} / \mathrm{kg}$ of ${ }^{99 \mathrm{~m}} \mathrm{Tc}$ labeled ECD was performed on subjects under resting conditions. The patients were lying with eyes closed in a quiet, dark or dimly lit environment from at least 10 minutes prior until 5 minutes post injection.

In order to acquire the ictal scans, video-EEG monitoring was used in order to monitor the onset of seizure. Injection of $10 \mathrm{MBq} / \mathrm{kg}$ of ${ }^{99 \mathrm{~m}} \mathrm{Tc}$ labeled ECD was performed immediately $(<40 \mathrm{sec})$ after seizure onset, either manually or with an injection pump.

For both ictal and inter-ictal image acquisition, the patients were lying down supine in the scanner, with their arms down. The image data was acquired on a Siemens MultiSPECT 3 scanner 30 minutes after injection of the tracer, with a scan duration of 30 minutes at most. The field of view of the image contained the entire brain and the cerebellum. The projection data was processed with filtered back projection, and Chang attenuation correction was applied. 


\section{B. Registration}

Since the ictal and inter-ictal SPECT dataset were acquired at different times, the ictal scan needs to be registered to the inter-ictal one prior to further processing. In a first step, both images are coarsely aligned using rigid registration. However, due to variations of the $\mathrm{CBF}$ in the ictal and interictal state which directly impacts the similarity measure, slight misregistrations would show up in the following processing steps and could be erroneously regarded as lesions. For this reason, a deformable registration is used in a second step. The deformable fine alignment is based on Gaussian radial basis functions [6], and uses mutual information [7] as objective function which is minimized during optimization. Extensive internal testing has shown that a deformable fine alignment provides slightly better results than affine registration. The registration quality was confirmed visually by a clinical expert (GP, co-author).

\section{Intensity Normalization}

In order to be compared to each other and to be subtracted as part of the asymmetry analysis, the ictal and inter-ictal SPECT scans require an intensity normalization. This is due to the varying time between injection and image acquisition, injection dose, as well as differences in tracer uptake, washout and decay. For these reasons, the intensities need to be normalized to a common intensity range. For this purpose, the mean brain pixel intensities of the ictal and inter-ictal SPECT images are normalized to a constant value.

\section{Standard Image Analysis}

For a comprehensive analysis of the data, different analysis methods were applied. First of all, reading by a nuclear medicine expert (GP, co-author) was performed for initial assessment. Subsequently, standard image analysis techniques commonly applied to ictal and inter-ictal SPECT datasets in epilepsy patients were performed:

1) Subtraction of Ictal and Inter-ictal SPECT: Since the epileptic focus features abnormally low perfusion in the interictal scan, and increased perfusion in the ictal scan, the difference image is expected to highlight this region [3]. In order to review the subtraction image in an anatomical context, it can be overlaid onto an MR scan, which is registered to the inter-ictal SPECT scan. This is denoted by subtraction ictal SPECT co-registered to MRI (SISCOM) [4].

2) Database Comparison: Comparison of a patient brain to a reference database is a common approach for analysis of nuclear medicine datasets and has also been used for PET and SPECT images in epilepsy patients [8], [2]. In this work, the reference database is obtained from averaging a set of 30 normal subjects (aged 16-49, mean age of 32), and comprises a volume of mean values $V_{\text {mean }}$ and a volume of standard deviations $V_{\text {stdDev }}$. For each voxel $i$, a Z -score is calculated which expresses the intensity deviation of the patient scan from the mean in terms of standard deviations:

$$
Z \text { score }(i)=\frac{V_{\text {patient }}(i)-V_{\text {mean }}(i)}{V_{\text {stdDev }}(i)}
$$

\section{E. Novel Method for Asymmetry Analysis}

During a seizure, unilateral activation of the focal region in the respective hemisphere is observed, which makes asymmetry analysis a useful tool for lesion detection. However, since the brain hemispheres are not perfectly symmetric, differences between the hemispheres also occur due to anatomical differences. Furthermore, functional asymmetries may occur resulting in asymmetric uptake on both hemispheres, which makes asymmetry analysis difficult.

For this reason, a novel approach for asymmetry analysis is presented in this work, which makes it possible to distinguish between anatomical or functional asymmetry in regions other than the epileptic focus, and asymmetry due to an epileptic lesion. The approach is outlined in Figure 1, and comprises the following steps:

1. Ictal asymmetry dataset: A left-right flipped version of the ictal dataset is computed and registered to the original ictal dataset. The ictal asymmetry dataset is then computed by subtracting the flipped ictal from the ictal dataset.

2. Inter-ictal asymmetry dataset: Similarly, a flipped version of the inter-ictal dataset is computed and registered to the inter-ictal dataset. The inter-ictal asymmetry dataset is then computed by subtracting the flipped inter-ictal from the inter-ictal dataset.

3. Total difference image: The inter-ictal asymmetry dataset (2.) is subtracted from the ictal asymmetry dataset (1.) to account for anatomical asymmetries.

On top of asymmetries due to an epileptic focus, the asymmetry images computed for both the ictal and inter-ictal scan also contain both anatomical and functional asymmetries. In order to suppress the unwanted anatomical and functional asymmetries, both asymmetry images are subtracted in order to obtain the final asymmetry image. In an ideal scenario, this image would show asymmetries due to the epileptic lesion only.

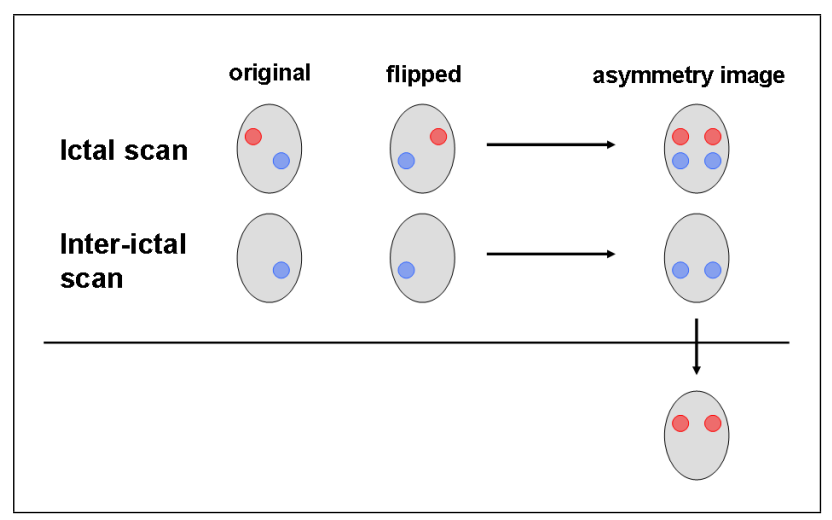

Fig. 1. Schematic outline of the novel asymmetry analysis approach. The red circle indicates the location of the focus, whereas the blue circle denotes an anatomical asymmetry. The ictal and inter-ictal asymmetry image (left) are flipped (middle) and subtracted (right) from the original image, resulting in an ictal and inter-ictal asymmetry image. In order to exclude anatomical asymmetries, both asymmetry images are subtracted (final result). 


\section{RESUlTS AND Discussion}

The standard image analysis methods as well as the novel approach for asymmetry analysis were applied to ictal and inter-ictal SPECT datasets in 10 patients. In all cases, anatomical MRI as well as EEG recordings were available as well, but were not fully conclusive.

Reading by a nuclear medicine expert, which incorporates anatomical knowledge and experience about likely positions of epileptic foci, is indispensable for the analysis of ictal and inter-ictal SPECT datasets. However, image processing methods such as subtraction, database comparison and asymmetry analysis can support this process and provide valuable information about the potential location of an epileptic focus.

In Figure 2, subtraction of the ictal and inter-ictal SPECT dataset are shown for two different patients (upper and lower row, respectively). For the first patient, variations in $\mathrm{CBF}$ are clearly visible in the subtraction image and make it possible to identify the location of the epileptic focus. However, this approach may not be conclusive in each case, as demonstrated for the second patient (lower row).
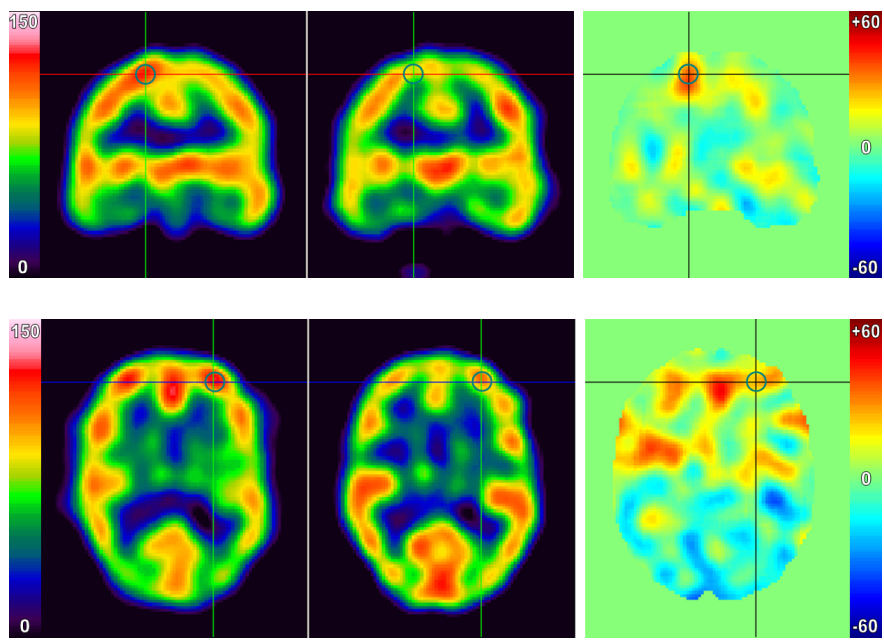

Fig. 2. Subtraction: Ictal (left), inter-ictal (middle) SPECT dataset and subtraction image (right)in two different patients (upper row, lower row). The circle indicates the location of the epilleptic focus. Upper row: In this patient, the subtraction image clearly shows the location of the epileptic focus. Lower row: In this patient, the subtraction image is not able to highlight the focus.

For the second patient, the approach for database comparison provides more significant results (Figure 3). Since the comparison based on Z-scores involves the standard deviation of the normal population, this method is very well suited to account for regions with highly varying uptake characteristics. In this patient, the epileptic focus is clearly visible on the Zscore map.

The approach for asymmetry analysis makes it possible to distinguish between asymmetry due to an epileptic lesion, and other anatomical or functional asymmetries as demonstrated in Figure 4. The filled arrows indicate functional asymmetry due to the epileptic focus, whereas empty arrows indicate other asymmetries. In the final asymmetry image (middle), some of the undesired asymmetries cancel out and disappear.

The propsed method for asymmetry analysis was able to show the focus in 8 out of the 10 patients under investigation.
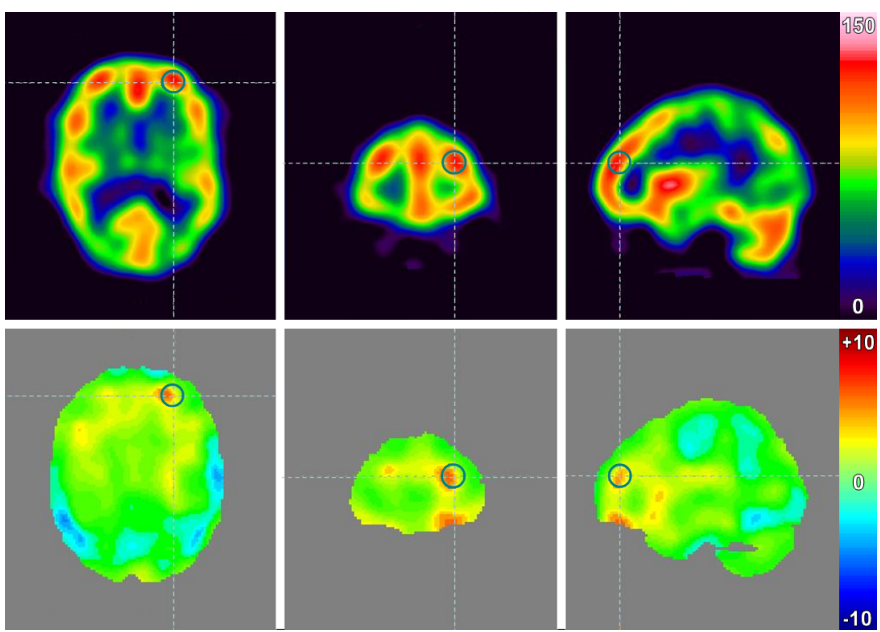

Fig. 3. Database comparison. The ictal SPECT image (upper row) is compared against a normal database, and z-score results are shown (lower row). The circle indicates the location of the epileptic focus.

However, in some patients functional asymmetries which are not associated with the epileptic focus are visible as well on the final image. This can be explained by different behaviour of the patient (movement, activation of visual cortex) after injection of the tracer, which leads to different functional asymmetries in the ictal and inter-ictal scan and to varying uptake patterns.

Overall, assessment of epilepsy patients is a difficult task and therefore requires multiple imaging, data analysis and assessment techniques. Along with other standard data analysis approaches, the novel asymmetry method for ictal and interictal SPECT datasets presented in this work is a valuable supplement to identify the location of epileptic foci and to support reading by a nuclear medicine expert. Nevertheless, reading and interpreting of the results needs profound neurological and functional knowledge in order to reliably identify the location of an epileptic focus.

\section{Conclusion}

In this work, an advanced approach for asymmetry analysis of ictal and inter-ictal SPECT data is presented. The appraoch addresses the problem of anatomical asymmetries showing up in the ictal asymmetry image by subtracting the inter-ictal asymmetry image from it. Overall, the suggested technique improves asymmetry analysis by reducing the number of lesions on the asymmetry image, and provides clinicans with an additional method of analysis in epilepsy patients.

\section{ACKNOWLEDGMENT}

This work is funded by Siemens Molecular Imaging, Oxford, UK. 

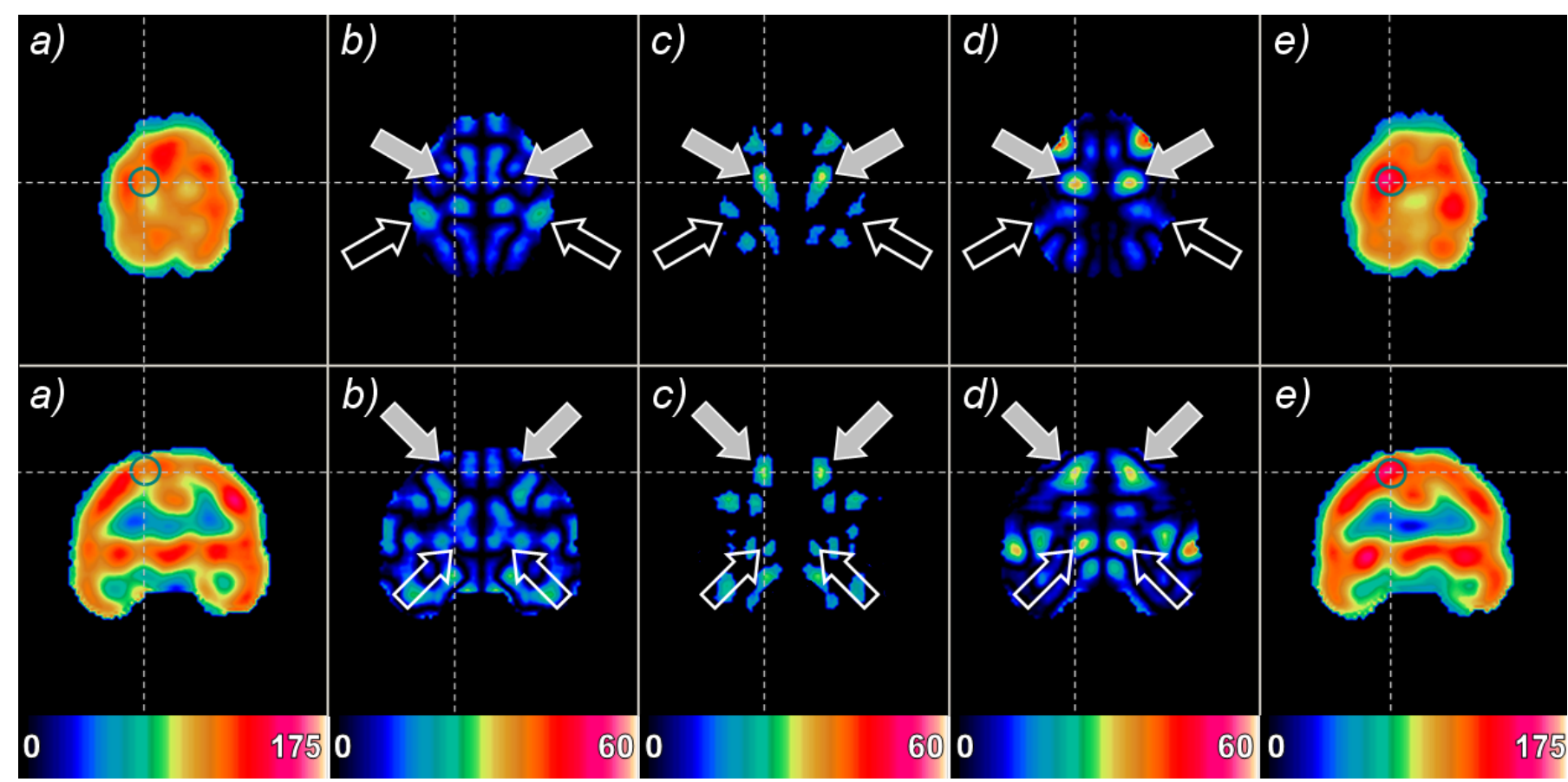

Fig. 4. Asymmetry analysis. The circle indicates the location of the focus in axial (upper row) and coronal (lower row) slices of the same patient. a) Inter-ictal scan. $e$ ) Ictal scan. $b$ ) Inter-ictal asymmetry image. $d$ ) Ictal asymmetry image. $c$ ) Subtraction of ictal and inter-ictal asymmetry image (final result).

\section{REFERENCES}

[1] Gruenwald, F., Menzel, C., Pavics, L., Bauer, J., Hufnagel, A., Reichmann, K., Sakowski, R., Elger, C., Biersack, H.: Ictal and interictal brain SPECT imaging in epilepsy using technetium-99m-ECD. J Nucl Med. 35 (1994) 1896-901

[2] Goffin, K., Dedeurwaerdere, S., Laere, K.V., Paesschen, W.V.: Neuronuclear assessment of patients with epilepsy. Semin Nucl Med. 38 (2008) 227-239

[3] Zubal, I., Spencer, S., Imam, K., Seibyl, J., Smitha, E., Wisniewski, G., Hoffer, P.: Difference images calculated from ictal and interictal technetium-99m-HMPAO SPECT scans of epilepsy. J Nucl Med. 36 (1995) 684-689

[4] O’Brien, T., So, E., Mullan, B., Hauser, M., Brinkmann, B., Bohnen, N., Hanson, D., Cascino, G., Jack, C., Sharbrough, F.: Subtraction ictal SPECT co-registered to MRI improves clinical usefulness of SPECT in localizing the surgical seizure focus. Neurology 50 (1998) 445-454

[5] Rowe, C., Berkovic, S., Austin, M., Saling, M., Kalnins, R., McKay, W., Bladin, P.: Visual and quantitative analysis of interictal SPECT with technetium-99m-HMPAO in temporal lobe epilepsy. J Nucl Med. 32 (1991) 1688-1694

[6] Arad, N., Dyn, N., Reisfeld, D., Yehezkel, Y.: Image warping by radial basis functions: applications to facial expressions. CVGIP-Graph Model Im 56 (1994) 161-172

[7] Wells, W., Viola, P., Atsumi, H., Nakajima, S., Kikinis, R.: Multi-modal volume registration by maximization of mutual information. IEEE Trans Med Imaging 1 (1996) 35-52

[8] Drzezga, A., Arnold, S., Minoshima, S., Noachtar, S., Szecsi, J., Winkler, P., Rmer, W., Tatsch, K., Weber, W., Bartenstein, P.: 18F-FDG PET studies in patients with extratemporal and temporal epilepsy: evaluation of an observer-independent analysis. J Nucl Med. 40 (1999) 737-746 